\title{
Traveling wavefront solutions to nonlinear reaction-diffusion-convection equations
}

\author{
Joseph O. Indekeu ${ }^{1}$ and Ruben Smets $^{1}$ \\ ${ }^{1}$ Institute for Theoretical Physics, KU Leuven, \\ Celestijnenlaan 200D, BE-3001 Leuven, Belgium
}

(Dated: May 31, 2017)

\begin{abstract}
Physically motivated modified Fisher equations are studied in which nonlinear convection and nonlinear diffusion is allowed for besides the usual growth and spread of a population. It is pointed out that in a large variety of cases separable functions in the form of exponentially decaying sharp wavefronts solve the differential equation exactly provided a co-moving point source or sink is active at the wavefront. The velocity dispersion and front steepness may differ from those of some previously studied exact smooth traveling wave solutions. For an extension of the reactiondiffusion-convection equation, featuring a memory effect in the form of a maturity delay for growth and spread, also smooth exact wavefront solutions are obtained. The stability of the solutions is verified analytically and numerically.
\end{abstract}

PACS numbers: 14.80.Va, 03.75.Lm, 03.75.Kk 


\section{INTRODUCTION}

Traveling wave solutions play an important role in the physical characterization of biological systems or other active matter [1], in modeling the growth and diffusion of a (human) population over large spatial scales and long times [2], and constitute a central theme in mathematical biology [3]. The search for exact wavefront solutions of differential equations that are modifications of the (one-dimensional) Fisher reaction-diffusion equation has a long history and has been quite active in recent years [4-7]. On the one hand, the existence of such solutions is a problem which has received much recent attention, and, on the other hand, it is important to establish under which conditions such solutions can be reached by time evolution from suitably chosen initial conditions $u(x, t=0)$ for the population density or other "concentration"-like fields $u(x, t)[8]$.

It is well-known that for the classical Fisher "model" system (without convection) no exact solutions have been obtained except for special values of the propagation velocity. Our goal in this note is to study physically motivated modified Fisher models for which exact solutions are more straightforwardly available. In particular, we point at the existence of a class of separable exponential tail solutions for models with a physical constraint imposed on the concentration at the wavefront. Some of the solutions we discuss display new physical characteristics. For example, for the convective Fisher model, contrary to what is commonly found for smooth wavefront solutions, the exponential tail solutions display a wavefront that steepens and sharpens as the strength of the convective correction is reduced to retrieve the classical Fisher model.

The solutions we mainly focus on are traveling wavefronts with a sharp exponential tail (see Fig.1a). They are examples of (weakly) singular functions that are physically acceptable. Other examples are so-called sharp edge solutions, which are likewise singular, and feature a sharp front (see Fig.1b). Profiles with stronger singularities (e.g., a discontinuity in the concentration) are not considered here, although they are still of interest biochemically or biophysically [3]. Smooth wavefronts are illustrated in Fig.1c.

The weakly singular (or "piecewise analytic") solutions we study are mathematically exact provided a source or sink term is added to the differential equation. This term is localized sharply about the traveling wavefront position. The (bio-)physical motivation of the source can be the local injection of nutrient or administration of stimulus by a co-moving 
probe, while the sink can correspond to a toxic component or mechanical device which kills or removes concentration at the front. Alternatively, one may think of the source/sink being caused by the presence of a second component or species, the concentration of which is confined to the "interface" defined as the zone of largest gradient of concentration of the spreading population. In this case, the population does not "back-react" on the second component and we neglect possible cross-diffusion such as studied, e.g., in [9]. Note that we have in mind sources or sinks of the concentration field itself, rather than considering sources or sinks as coherent structures in which wavefronts are, respectively, generated or absorbed (in oppositely traveling pairs) as studied, e.g., in [10].

Mathematically, we consider this source/sink term to be the delta-function limit of a (Gaussian) profile with a width small compared to the decay length (in case of a tail) or recovery length (in case of an edge) of the wavefront. One may be tempted to speculate that the solutions with delta-function sources or sinks can lead to a Green's function approach with which solutions with spatially more extended smooth sources or sinks can be constructed using superposition. However, this is in general not possible in view of the nonlinear character of the differential equation.

The paper is organized as follows. In Section II we develop the first differential equation for which we discuss exact sharp exponential tail solutions, the Fisher equation with a nonlinear convective term. We show that these solutions are complementary to those found by Mishra and Kumar [6] and display a different wave velocity dispersion. In Section III we study models with concentration-dependent diffusion. We first point out that a combination of gradient-dependent growth and a concentration-dependent diffusion coefficient can lead to a simple differential equation which is again solved by sharp exponential tails. Next we illustrate the relevance of our solutions for the standard (but nontrivial) model with concentration-dependent diffusion. Section IV presents a more general class of nonlinear reaction-diffusion-convection equations, including a time delay between stimulus and growth. Also these models possess sharp exponential tail solutions and sharp edge solutions. We compare our models and results with those of others and also offer a particular smooth wavefront solution. In Section $V$ the windows in parameter space for the stability of the solutions are established analytically and verified numerically. Conclusions are drawn in Section VI. 


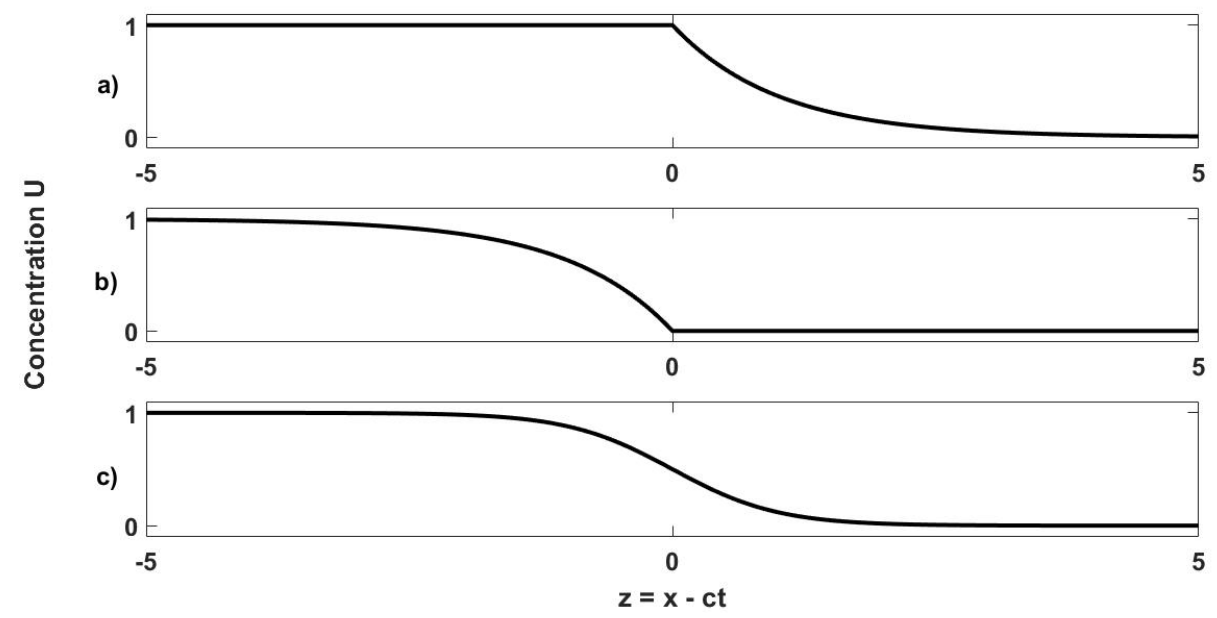

FIG. 1. Traveling wave solutions $U(z)$ of modified Fisher equations. (a): a sharp exponential tail solution (e.g., $U(z)=\exp (-z)$ for $z>0$ ), (b): a sharp edge solution (e.g., $U(z)=1-\exp (z)$ for $z<0)$, (c): a smooth solution (e.g., $U(z)=(1-\tanh (z)) / 2)$.

\section{CONVECTIVE FISHER EQUATION}

We recall the classical Fisher equation [11],

$$
\frac{\partial u}{\partial t}=\alpha u(1-u)+D \frac{\partial^{2} u}{\partial x^{2}}
$$

which describes the growth of the (dimensionless) concentration $u(x, t)$ to a certain carrying capacity (here normalized to 1) and the concommittant diffusive motion. The growth rate is $\alpha$ and the diffusion constant is $D$. These constants can be removed by the transformation: $t \leftarrow \alpha t, x \leftarrow \sqrt{\alpha / D} x$. We thus arrive at

$$
\frac{\partial u}{\partial t}=u(1-u)+\frac{\partial^{2} u}{\partial x^{2}}
$$

The following generalization of the one-dimensional Fisher equation has been proposed to allow for various kinds of convective motion [3],

$$
\frac{\partial u}{\partial t}+\frac{\partial h(u)}{\partial x}=u(1-u)+\frac{\partial^{2} u}{\partial x^{2}}
$$

For the function $h(u)$ it is natural to consider a second-order polynomial, $h(u)=a_{0}+a_{1} u+$ $a_{2} u^{2}$. The constant is irrelevant and the first-order term represents a constant convective velocity, which usually amounts just to a simple shift in the propagation velocity. Therefore, 
focusing on a non-trivial second-order effect through $h(u)=k u^{2} / 2[3,6,7]$, where $k$ is a positive dimensionless constant, one obtains the following specific form,

$$
\frac{\partial u}{\partial t}+k u \frac{\partial u}{\partial x}=u(1-u)+\frac{\partial^{2} u}{\partial x^{2}}
$$

Adding a delta-source term in the co-moving frame,

$$
\frac{\partial u}{\partial t}+k u \frac{\partial u}{\partial x}=u(1-u)+\frac{\partial^{2} u}{\partial x^{2}}+\frac{1}{k} \delta(x-c(k) t)
$$

where the parameter function $c(k)$ must be chosen to match the propagation velocity selfconsistently, we observe that the differential equation possesses the following traveling wave solution with an exponential tail,

$$
u(x, t)=U(z)= \begin{cases}1, & z<0 \\ e^{-\frac{z}{k}}, & z \geq 0\end{cases}
$$

where $z=x-c t$, with $c$ the velocity of the traveling wave, and $k$ is required to be positive to provide a physical solution. The front of the wave is conveniently chosen to start decaying from $z=0$. It is readily verified that (6) solves (5) when the following dispersion relation is met,

$$
c(k)=k+\frac{1}{k}
$$

relating the velocity of the wave to the steepness of the front or, equivalently in this case, the decay constant of the tail. We remark that the amplitude of the delta-function point source is determined by integrating the differential equation, expressed in the co-moving frame, from $z=0^{-}$to $z=0^{+}$and requiring that the result be an identity.

Rather than by trial and error, we derive this solution more systematically by attempting separation of variables. Assuming the existence of a separable solution, $u(x, t)=X(x) T(t)$, we are led to

$$
\frac{T^{\prime}}{T}+\left(k X^{\prime}+X\right) T=1+\frac{X^{\prime \prime}}{X}
$$

which indicates that a product form can be found provided the expression between the parentheses is a constant. Further inspection shows that this constant must be zero. This leads us to (6).

Murray and Gibbs studied this model (without point source or sink) and the following 
exact lower bound for the wave velocity was obtained [3, 12],

$$
c_{\ell}(k)= \begin{cases}2, & 2>k>0 \\ \frac{k}{2}+\frac{2}{k}, & 2 \leq k<\infty,\end{cases}
$$

and it has also been known since long that a solution of Eq.(4) exists for any wave velocity $c \geq c_{\ell}(k)$. It is easily seen that $k+\frac{1}{k} \geq 2$ for $0<k<2$ and that $k+\frac{1}{k} \geq \frac{k}{2}+\frac{2}{k}$ for $k>2$ so that the solution we present satisfies this lower bound property of the wave velocity.

It is interesting to compare result (6) with the exact smooth solution found by Mishra and Kumar [6],

$$
U_{\mathrm{MK}}(z)=\frac{1}{2}\left(1-\tanh \left(\frac{k z}{4}\right)\right)
$$

with wave velocity [7]

$$
c_{\mathrm{MK}}(k)=\frac{k}{2}+\frac{2}{k}
$$

which clearly differs from the wave velocity (7) found for (6). For $k<\sqrt{2}$, solution (10) has a greater wave velocity, while for $k>\sqrt{2}$, solution (6) has a greater wave velocity. When $k=\sqrt{2}$, the intersection point of the dashed and dotted curves in Fig.2, both solutions have the same wave velocity. Furthermore, since $1 / k$ then equals $k / 2$, they have the same steepness. The steepness of the wave is defined as the derivative of the solution with respect to $z$ at $z=0$ (where the derivative is maximal in absolute value). Note that then also the decay constants for $z \rightarrow \infty$ coincide for both solutions. This could have been expected since the wave velocity is related to the asymptotics of the tail $[3,8]$.

An interesting observation is that the parameter $k$ is inversely proportional to the steepness of the wavefront for the solution (6) presented here, whereas it is directly proportional to the steepness for the solution found by Mishra and Kumar (10). As a consequence, for our solutions the front sharpens to a step in the Fisher model limit $k \rightarrow 0$.

In closing this section we note that (4) possesses edge solutions for $k<0$, which become exact when a suitable delta-sink is added. These solutions are of the form

$$
u(x, t)=U(z)= \begin{cases}1-\exp (\alpha z), & z<0 \\ 0, & z \geq 0,\end{cases}
$$

with necessarily $\alpha>0$ ( $\alpha^{-1}$ being the recovery length) and with $z=x-c t$. However, it is 


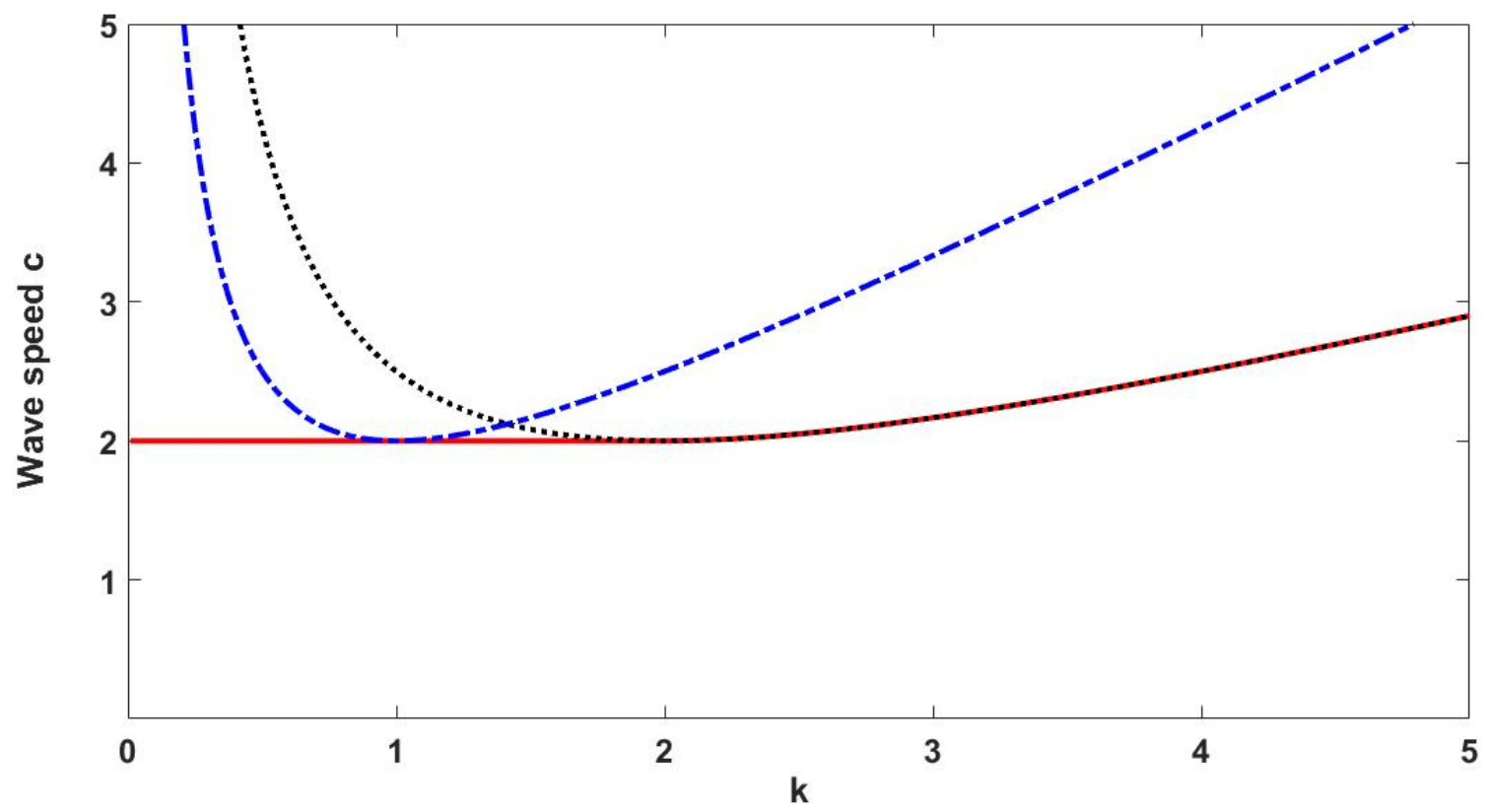

FIG. 2. Wave velocity $c$ versus nonlinear convection amplitude $k$ for different solutions of Eq.(4). Blue, dashed line: wave velocity of the proposed solution (6); black, dotted line: wave velocity of the solution (10) given by Mishra and Kumar; red, solid line: exact lower bound (9).

easily verified that these solutions are characterized by $\alpha=-1 / k>0$, and by $c=1 / k<0$, corresponding to a receding concentration profile (wavefront traveling towards the left).

\section{CONCENTRATION-DEPENDENT DIFFUSION}

\section{A. Effect of gradient-dependent growth}

The classical Fisher equation is a special case of the following general reaction-diffusion equation

$$
\frac{\partial u}{\partial t}=f\left(u, \frac{\partial u}{\partial x}\right)-\frac{\partial}{\partial x} J\left(u, \frac{\partial u}{\partial x}\right)
$$

where $f$ is a growth function and $J$ some current which is conserved when the growth function vanishes, typically of the form $J=-D(u) \partial u / \partial x$ with $D(u)$ the (possibly) concentrationdependent diffusion coefficient. The growth function in the classical Fisher equation depends only on the concentration, but it makes sense physically to include also a gradient term in the growth function. The motivation for this is that the growth (of bacteria, for example) 
at some point in space is not only influenced by the concentration at that point, but also by the nearby concentration. A possible form for such a growth function is the following,

$$
f\left(u, \frac{\partial u}{\partial x}\right)=u(1-u)-k^{2}\left(\frac{\partial u}{\partial x}\right)^{2}
$$

It consists of logistic growth supplemented with a gradient-squared term which suppresses the growth depending on the concentration inhomogeneity. The second power of the gradient ensures the physical symmetry that not the sign but only the magnitude of the gradient matters. Following the approach of Murray [3], the diffusion coefficient is chosen such that it varies linearly with the concentration. The specific form used here is $D(u)=1+k^{2} u$. Inserting this growth function and diffusion coefficient in (13) yields the following dynamical equation,

$$
\frac{\partial u}{\partial t}=u(1-u)+\left(1+k^{2} u\right) \frac{\partial^{2} u}{\partial x^{2}}
$$

which we endow with a point source as follows,

$$
\frac{\partial u}{\partial t}=u(1-u)+\left(1+k^{2} u\right) \frac{\partial^{2} u}{\partial x^{2}}+c(k) \delta(x-c(k) t)
$$

We observe that the earlier proposed solution (6) also solves (16) with the same dispersion relation (7).

One can further generalize the dynamical equations (4) and (15) for which (6) provides a piecewise solution to

$$
\frac{\partial u}{\partial t}=u(1-u)+\frac{\partial^{2} u}{\partial x^{2}}+(-k)^{m} u \frac{\partial^{m} u}{\partial x^{m}}
$$

where $m \in \mathbb{N}^{+}$. For all values of $m$, dispersion relation (7) is recovered when (6) is inserted in equation (17). However, for $m>2$, the solution cannot be rendered exact at $z=0$ by adding a (simple) delta-function source term.

\section{B. Standard concentration-dependent diffusion}

In this section we revisit the model with concentration-dependent diffusion and show that the standard version of this problem leads to a differential equation which is slightly more involved than the examples considered so far. We again start from (13), but now with the standard growth function,

$$
\frac{\partial u}{\partial t}=u(1-u)+\frac{\partial}{\partial x}\left(D(u) \frac{\partial u}{\partial x}\right)
$$


with the choice

$$
D(u)=1+\frac{k^{2}}{2} u
$$

This leads to a slightly extended equation which contains also a gradient-squared term, and which, suitably decorated with a point source, becomes

$$
\frac{\partial u}{\partial t}=u(1-u)+\frac{k^{2}}{2}\left(\frac{\partial u}{\partial x}\right)^{2}+\left(1+\frac{k^{2}}{2} u\right) \frac{\partial^{2} u}{\partial x^{2}}+\left(\frac{k}{2}+\frac{1}{k}\right) \delta(x-c(k) t)
$$

Remarkably, the presence of this extra gradient-squared term does not spoil the simplicity of obtaining an exact wavefront solution and we observe that the simple sharp tail (6) also solves this equation, and again with velocity dispersion (7). In fact, the model can be extended considerably while still possessing exact exponential tail solutions, as will be shown in the next Section.

In closing this Section we note that the standard concentration-dependent diffusion model possesses also exact edge solutions with remarkable properties. These solutions require a point-sink term of the form $-\frac{1}{k} \delta(x-c(k) t)$ to be added to the differential equation. The edge solution (12) then applies with $\alpha=1 / k$ and the velocity is $c(k)=\left(k^{2}-2\right) /(2 k)$, with $k>0$. Surprisingly, the propagation velocity passes through zero continuously at $k=\sqrt{2}$ so that left-traveling, right-traveling and stand-still edge solutions can be obtained.

\section{EXTENSIONS OF THE MODEL}

Consider the following fairly general nonlinear reaction-diffusion-convection equation,

$$
\left(1+\tau f^{\prime}(u)\right) \frac{\partial u}{\partial t}+\left(\lambda_{c}+k_{c} u\right) \frac{\partial u}{\partial x}=f(u)+\lambda_{g}\left(\frac{\partial u}{\partial x}\right)^{2}+\left(\lambda_{d}+k_{d} u\right) \frac{\partial^{2} u}{\partial x^{2}},
$$

where the free parameters are all assumed to be non-negative. This equation slightly extends the class of equations considered by Cherniha and Dutka [13] in that we include a memory effect at the level of the growth function $f(u)$, which we evaluate at time $t-\tau$ instead of time $t$, so that there is, e.g., a small maturation delay before members of a population can spread or grow. The replacement is

$$
f(u(x, t)) \rightarrow f(u(x, t-\tau))=f(u(x, t))-\tau f^{\prime}(u) \frac{\partial u}{\partial t}
$$

This is reminiscent of, but different from, what is done in the commonly studied "telegraph" differential equations featuring more terms that are delayed (or advanced) in this manner. 
Those involve also a second derivative with respect to time [14-18]. As before, we consider models with $f(u)=u(1-u)$. Without loss of generality, we can rescale time by a factor $1+\tau$ so that our dynamical equation becomes, point-source inclusive,

$$
\left(1-k_{t} u\right) \frac{\partial u}{\partial t}+\left(\lambda_{c}+k_{c} u\right) \frac{\partial u}{\partial x}=u(1-u)+\lambda_{g}\left(\frac{\partial u}{\partial x}\right)^{2}+\left(\lambda_{d}+k_{d} u\right) \frac{\partial^{2} u}{\partial x^{2}}+\alpha\left(\lambda_{d}+k_{d}\right) \delta(x-c t),
$$

with $0 \leq k_{t}<1$ and with $\alpha$ and $c$ to be chosen self-consistently according to the following results.

Note that, unlike in the procedure followed in [13], we do not assume additional generating conditions. Our heuristic method for finding exact solutions is again to attempt separation of variables, i.e., $u(x, t)=X(x) T(t)$. This leads us to conclude that separable solutions can be found using the main equation

$$
\frac{T^{\prime}}{T}-1=\lambda_{d} \frac{X^{\prime \prime}}{X}-\lambda_{c} \frac{X^{\prime}}{X}
$$

provided the solutions satisfy the auxiliary equation

$$
-k_{t} \frac{T^{\prime}}{T}+1=k_{d} \frac{X^{\prime \prime}}{X}+\lambda_{g}\left(\frac{X^{\prime}}{X}\right)^{2}-k_{c} \frac{X^{\prime}}{X}
$$

If we denote the propagation velocity by $c$, we can write the solutions $X(x)$ and $T(t)$ formally as

$$
\begin{gathered}
X(x)=e^{-\alpha x} \\
T(t)=e^{\alpha c t}
\end{gathered}
$$

so that, with $z=x-c t$, the separable solution $u(x, t)=U(z)=\exp (-\alpha z)$ provides us with an exact wavefront solution for $z>0$, while $U(z)=1$ for $z<0$.

Substitution in (25) leads to

$$
\left(k_{d}+\lambda_{g}\right) \alpha^{2}+\left(k_{c}+k_{t} c\right) \alpha-1=0
$$

On the other hand, substitution of the proposed solution in (24) leads to

$$
\lambda_{d} \alpha^{2}+\left(\lambda_{c}-c\right) \alpha+1=0
$$

Combining these we obtain the (positive) decay constant,

$$
\alpha=\frac{-\left(k_{c}+\lambda_{c} k_{t}\right)+\sqrt{\left(k_{c}+\lambda_{c} k_{t}\right)^{2}+4\left(1-k_{t}\right)\left(k_{d}+\lambda_{g}+\lambda_{d} k_{t}\right)}}{2\left(k_{d}+\lambda_{g}+\lambda_{d} k_{t}\right)}
$$


The velocity follows immediately from (29),

$$
c_{t a i l}-\lambda_{c}=\frac{1}{\alpha}+\lambda_{d} \alpha
$$

and we observe that $\lambda_{c}$ not only causes a velocity shift relative to the co-convecting velocity, but also causes a modification of the spatial decay constant $\alpha$ through the presence of the product $k_{t} \lambda_{c}$. Therefore, in general we cannot ignore $\lambda_{c}$, although Cherniha and Dutka could safely ignore it in [13] since $k_{t}=0$ in their class of models. Incidentally, an exact exponential tail solution in accord with (30) was derived in [13] (see their Eq.10) for the case $\lambda_{c}=k_{c}=k_{t}=0, \lambda_{d}=1$ and $k_{d}=\lambda_{g} \equiv \lambda_{0}$.

It is interesting to study also possible exact sharp edge solutions of (23). Edge solutions are not separable, but it is straightforward to test an edge solution of the form (12). This leads to the decay constant

$$
\alpha=\frac{\left(k_{c}+\lambda_{c} k_{t}\right)+\sqrt{\left(k_{c}+\lambda_{c} k_{t}\right)^{2}+4\left(k_{d}+\lambda_{g}+\left(\lambda_{d}-\lambda_{g}\right) k_{t}\right)}}{2\left(k_{d}+\lambda_{g}+\left(\lambda_{d}-\lambda_{g}\right) k_{t}\right)}
$$

and velocity

$$
c_{e d g e}-\lambda_{c}=\alpha\left(\lambda_{g}-\lambda_{d}\right)
$$

Note that in order for the edge velocity to be positive for any $\lambda_{c} \geq 0$, the rather stringent requirement $\lambda_{g}>\lambda_{d}$ must be satisfied. Mansour [4] already obtained an exact edge solution for this problem, in the special case $k_{t}=\lambda_{c}=\lambda_{d}=0$ and $\lambda_{g}=k_{d}$. Also note that in this case a point-sink of the form $-\alpha \lambda_{d} \delta(x-c t)$ must be added to the differential equation.

Let us elaborate more on some interesting problems that can be described by (21). Firstly, we distinguish between problems with a finite diffusion coefficient at low concentration, $D(0) \neq 0$ and problems with $D(0)=0$. In the former case we can fix the spatial length scale through $\lambda_{d}=1$, without loss of generality. The problems we discussed in Sections I-III are all of this type, and we retrieve all our previous solutions.

Next, we address the case $D(0)=0$ and focus on the interesting case of nonlinear diffusion described by $D(u)=u$, also termed degenerate diffusion, studied in [3, 19] and introduced by Gurney and Nisbet [20]. In our notation this model corresponds to $k_{t}=\lambda_{c}=k_{c}=\lambda_{d}=0$ and $\lambda_{g}=k_{d}=1$, which corresponds to the familiar differential equation

$$
\frac{\partial u}{\partial t}=u(1-u)+\left(\frac{\partial u}{\partial x}\right)^{2}+u \frac{\partial^{2} u}{\partial x^{2}}
$$


Although sharp exponential tail initial conditions $u(x, 0)$ were used to study the time evolution of profiles towards wavefront solutions [19], the fact that there exist exact sharp exponential tail solutions $U(z)$ seems not to have been reported. They become mathematically exact provided a point-source of the form $\alpha \delta(x-c(k) t)$ is added to the differential equation. For this case we find $\alpha=\frac{1}{\sqrt{2}}$ and velocity $c_{\text {tail }}=\sqrt{2}$, so that the exact sharp exponential tail solution is given by

$$
U(z)= \begin{cases}1, & z<0 \\ e^{-\frac{x}{\sqrt{2}}-t}, & z \geq 0,\end{cases}
$$

This solution complements the sharp edge solution, usually presented for this problem [3],

$$
U(z)= \begin{cases}1-e^{\frac{x}{\sqrt{2}}-\frac{t}{2}}, & z<0 \\ 0, & z \geq 0,\end{cases}
$$

which, surprisingly, has a lower velocity $c_{\text {edge }}=1 / \sqrt{2}$. This effect is robust. If we assume $D(u)=k_{d} u$, the spatial coordinate simply gets rescaled and $\alpha$ as well as the velocities acquire an extra factor $\sqrt{k_{d}}$ so that the velocity of the edge solution is always a factor of 2 below that of the tail solution. There is another surprise. In order to render the sharp edge solution mathematically exact, including at $z=0$, no point sink term appears necessary. Indeed, the amplitude of the delta-function sink is zero, which can be traced to be due to the vanishing of the prefactor $u$ of the second derivative of $u$, at $z=0$, in the differential equation (34). For this particular solution we have not performed a comprehensive stability study.

We close this section by presenting an exact smooth solution for the following model,

$$
\left(1-k_{t} u\right) \frac{\partial u}{\partial t}+\left(\lambda_{c}+k_{c} u\right) \frac{\partial u}{\partial x}=u(1-u)+\frac{\partial^{2} u}{\partial x^{2}},
$$

The smooth wavefront solution of the familiar form

$$
U(z)=\frac{1}{2}(1-\tanh \alpha z),
$$

with $z=x-c t$, solves the differential equation provided

$$
c-\lambda_{c}=\frac{1}{2 \alpha}+2 \alpha,
$$


with

$$
\alpha=\frac{\left(k_{c}+\lambda_{c} k_{t}\right)+\sqrt{\left(k_{c}+\lambda_{c} k_{t}\right)^{2}+8 k_{t}\left(1-\frac{k_{t}}{2}\right)}}{8\left(1-\frac{k_{t}}{2}\right)}
$$

Firstly, note that, for $k_{t} \rightarrow 0$, we obtain $\alpha \rightarrow k_{c} / 4$ so that we retrieve the exact smooth solution of Mishra and Kumar for the equation without memory effects [6]. Further, as regards the memory effects, Mishra and Kumar solved a problem different from ours, corresponding to a telegraph Fisher equation with an additional second derivative with respect to time, and obtained exact smooth wavefront solutions [7]. In their model the coefficient of the second derivative with respect to time is also proportional to $k_{t}$ (in our notation) so that two memory terms are coupled. As a consequence, in their model the decay coefficient $\alpha$ depends crucially on the convective coefficient $k_{c}$ so that $\alpha$ vanishes for $k_{c} \rightarrow 0$. In contrast, for $k_{c}=\lambda_{c}=0$ we retain a non-trivial solution with a marked dependence on the memory effect,

$$
\alpha=\frac{1}{2} \sqrt{\frac{\frac{k_{t}}{2}}{1-\frac{k_{t}}{2}}},
$$

and a velocity dispersion determined through (39).

\section{STABILITY OF THE SOLUTIONS}

The stability of the solutions presented here was studied both analytically and numerically. The approach used for the analytical stability analysis is similar to the one used by Murray [3] and Duan [21]. A perturbation $v$ in the moving frame is applied to the solution at $t=0$, resulting in $V(z, t) \equiv U(z)+\epsilon v(z, t)$, with $\epsilon \ll 1$. Upon insertion in the dynamical equations, a linear differential equation is found which is solved by the perturbation $v(z, t)$. Using Sturm-Liouville theory, sufficient conditions for stability of the solutions are obtained.

The result is that for the convective Fisher equation (4), the tail solution is stable for $k>0$, the edge solution is stable for $-1 / 2 \leq k<0$ and the smooth solution is stable for $|k|<8^{1 / 4}$. For the Fisher equation with gradient-dependent growth (15), as well as for the Fisher equation with concentration-dependent diffusion (20), the tail solution is stable for all positive $k$ and the edge solution is stable for $0<k \leq 1 / 2$.

These results were verified numerically using Matlab's partial differential equation method pdepe, which was also used in [2]. This method uses the ODE15s solver to advance the solution forward in time. This is a quasi-constant step size implementation of Klopfenstein's 
numerical differentiation formulae [22] in terms of backward differences [23]. To implement the localized source (or sink) either an interface condition $v(z=0, t)=0$ was imposed, or a Gaussian source (or sink) function of finite width was employed. The simulations show that the initially perturbed solutions (where both smooth and random perturbations were used) converge towards the analytical solutions in accordance with the theoretical predictions. Indeed, the tail solution proves to be stable for all positive $k$ for all three dynamical equations considered. For the DE (4), the edge solution becomes abruptly unstable for $k<-1 / 2$ as the perturbations cause outgrowth in the regime $z>0$. The smooth solution is stable for $|k|<8^{1 / 4}$. When $|k|$ exceeds that value, the solution becomes gradually unstable. For both DE's (15) and (20), the edge solution is stable for $0<k \leq 1 / 2$. The solution becomes abruptly unstable for $k>1 / 2$.

\section{CONCLUSION}

It has been shown that a variety of physically motivated Fisher-like reaction-diffusion equations equiped with a convective term possess exact sharp exponential tail (or sharp edge) wavefront solutions that have hitherto not received much attention. These solutions, relevant for modeling population dynamics, can be made mathematically exact on the entire domain by adding a delta-function source or sink at the interface formed by the traveling wavefront. This addition can be justified on physical grounds by considering the action of an external probe or the presence of a second species that interacts with the concentration

of the growing and spreading population. Some of these solutions evolve towards step function profiles in the limit that the classical Fisher equation is recovered. In a reactiondiffusion-convection model, for which simple exact smooth wavefront solutions were found in previous work, the exponential tail solution provides an even simpler alternative in the form of a separable function and with a different velocity dispersion relation.

The proposed type of solution also fits other generalizations of Fisher-type equations that describe biophysical or biochemical systems. A variety of such generalizations has been proposed and exact sharp exponential tail (or sharp edge) solutions as well as exact smooth solutions have been derived for selected examples, notably for a model featuring a memory effect corresponding to a maturity delay for growth and spread. The stability of the solutions 
to small perturbations has been verified by means of analytical calculations and numerical computations. An important next step in this research would be to investigate which initial conditions $u(x, 0)$ evolve, asymptotically for long times, towards the traveling wavefronts obtained here.

[1] G. De Magistris and D. Marenduzzo, An introduction to the physics of active matter, Physica A 34, 65 (2015)

[2] W.P. Petersen, S. Callegari, G.R. Lake, N. Tkachenko, J.D. Weismann, CH.P.E. Zollikofer, $A$ stable finite-difference scheme for population growth and diffusion on a map, PLoS One 12(1): e0167514 (2017)

[3] J.D. Murray, Mathematical biology: I. An Introduction, Third Edition, Springer (2002)

[4] M. B. A. Mansour, Traveling wave solutions for the extended Fisher/KPP equation, Rep. Math. Phys. 66, 375 (2010)

[5] W. Yuan, Q. Chen, J. Qi, Y. Li, The general traveling wave solutions of the Fisher equation with degree three, Adv. Math. Phys. 657918 (2013)

[6] A. Mishra, R. Kumar, Exact solutions of variable coefficient nonlinear diffusionreaction equations with a nonlinear convective term, Phys. Lett. A 374, 2921 (2010)

[7] A. Mishra, R. Kumar, Memory effects in Fisher equation with nonlinear convection term, Phys. Lett. A 376, 1833 (2012)

[8] D.A. Larson, Transient bounds and time-asymptotic behavior of solutions to nonlinear equations of Fisher type, SIAM J. Appl. Math. 34, 93 (1978)

[9] G. Gambino, M.C. Lombardo, M. Sammartino, Turing instability and traveling fronts for a nonlinear reaction-diffusion system with cross-diffusion, Mathematics and Computers in Simulation 82, $1112(2012)$

[10] R. Alvarez, M. van Hecke, W. van Saarloos, Sources ad sinks separating domains of left-and right-traveling waves: experiment versus amplitude equations, Phys. Rev. E 56, R1306 (1997)

[11] R.A. Fisher, The advance of advantageous genes, Ann. of Eugenics 7, 355 (1937)

[12] J.D. Murray, Nonlinear differential equation models in biology, Oxford: Clarendon Press (1977).

[13] R. Cherniha, V. Dutka, Exact and numerical solutions of the generalized Fisher equation, Rep. 
Math. Phys. 47, 393 (2001)

[14] C. Cattaneo, Sur une forme de l'équation éliminant le paradoxe d'une propagation instantanée, C. r. hebd. scéanc. Acad. sci. (Paris) 247, 431 (1958)

[15] H.A. Abdusalam, Analytic and approximate solutions for Nagumo telegraph reaction diffusion equation, Applied Mathematics and Computation 157, 515 (2004)

[16] H.I. Abdel-Gawad, A model for a finite memory transport in the Fisher equation, Applied Mathematical Modeling 32, 1883 (2008)

[17] H.A. Abdusalam, E.S. Fahmy, Exact solution for the generalized Telegraph Fisher's equation, Chaos, Solitons and Fractals 41, 1550 (2009)

[18] R.A. Van Gorder, K. Vajravelu, Analytical and numerical solutions of the density dependent Nagumo telegraph equation, Nonlinear Analysis: Real World Applications 11, 3923 (2010)

[19] J.A. Sherratt, B.P. Marchant, Nonsharp travelling wave fronts in the Fisher equation with degenerate nonlinear diffusion, Appl. Math. Lett. 9, 33 (1996)

[20] W.S.C. Gurney, R.M. Nisbet, The regulation of inhomogeneous population, J. Theor. Biol. 52, 441 (1975); ibid., A note on nonlinear population transport, J. Theor. Biol. 56, 249 (1976)

[21] W.-S. Duan, H.-J. Yang, Y.-R. Shi, An exact solution of Fisher equation and its stability, Chinese Physics 15, 1414 (2006)

[22] R.W. Klopfenstein, Numerical differentiation formulas for stiff systems of ordinary differential equations, RCA Rev. 32, 447 (1971)

[23] L. F. Shampine, M. W. Reichelt, The Matlab ODE suite, SIAM J. Sci. Comput., 18, 1 (1997) 